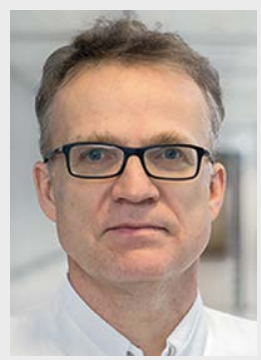

Gernot Keyßer

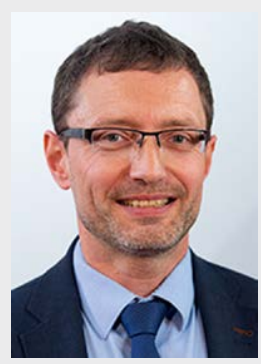

Eugen Feist

\section{Danke für 12 Jahre Engagement}

Liebe Leserinnen und Leser der „Aktuellen Rheumatologie“,

mit dem Ende des Jahres 2020 endet auch die Mitherausgeberschaft von Prof. Dr. med. Dr. med. habil. Stefan Rehart. Professor Rehart war seit 2008 Herausgeber der „Aktuellen Rheumatologie" und hat in dieser Zeit mit bewundernswerter Energie und Kreativität den Kurs dieser Zeitschrift mitbestimmt. Neben seiner anspruchsvollen Tätigkeit als Chefarzt der Klinik für Orthopädie und Unfallchirurgie am Agaplesion Markus-Krankenhaus in Frankfurt a. M. hat er sich immer wieder Zeit genommen, um dem Journal wertvolle Impulse zu verleihen. So war er seit 2004 Autor oder Mitautor von nicht weniger als 27 Artikeln in unserer Zeitschrift. Ebenso aktiv war er als Gutachter sowie als Initiator und Betreuer von insgesamt 12 Schwerpunktheften.

In die Periode seiner Herausgeberschaft fällt seine Präsidentschaft der Deutschen Gesellschaft für orthopädische Rheumatologie (DGORh), die er von 2010 bis 2016 innehatte. In dieser Zeit konnte er maßgeblich die gute und fruchtbringende Kommunikation der DGORh mit der stärker internistisch geprägten Deutschen Gesellschaft für Rheumatologie (DGRh) prägen. Diese Tatsache verdeutlicht die tiefe Verbundenheit von Prof. Rehart mit der Rheumatologie und zeigt, wie ernst es ihm um die Verknüpfung von rheumatologischer Orthopädie und internistischer Rheumatologie ist. Sein Anliegen hat seine Tätigkeit für die „Aktuelle Rheumatologie“ maßgeblich mitbestimmt.

Seine Begabung für die wissenschaftliche Publizistik äußert sich auch in der Tatsache, dass er neben der „Aktuellen Rheumatologie“ über viele Jahre die Zeitschriften „Arthritis und Rheuma“ sowie „Der Orthopäde“ als Mitherausgeber betreut hat und in zahlreichen weiteren Fachjournalen als Beirat aktiv war. Darüber hinaus ist er Autor einer ganzen Reihe von Buchbeiträgen, bei denen die Rheumachirurgie einen Schwerpunkt bildet.

Seine herausragende berufliche Qualifikation hat in zahlreichen Ehrungen und Berufungen Niederschlag gefunden. Zu nennen sei nur die ununterbrochene Nennung in der Fokus-Ärzteliste zwischen 2010 und 2020 sowie die 2019 erfolgte Berufung in den Kernvorstand der DGOOC.

Wir sind stolz darauf, mit Prof. Rehart über Jahre zusammenarbeiten zu dürfen und bedanken uns sehr herzlich für die produktive, stets zuverlässige und überaus qualifizierte Arbeit als Herausgeber der „Aktuellen Rheumatologie“.

Gernot Keyßer

Eugen Feist 